\title{
Quantitative Mapping of Strain, Polarization, and Octahedral Distortion at unit cell resolution by Scanning Electron Diffraction
}

\author{
Jim Ciston ${ }^{1}$, Roberto dos Reis ${ }^{1}$, Yifei Meng ${ }^{1}$, Colin Ophus ${ }^{1}$, and Lane Martin ${ }^{2}$ \\ 1. National Center for Electron Microscopy, Molecular Foundry, Lawrence Berkeley National \\ Laboratory, Berkeley, CA, USA \\ 2. Department of Materials Science and Engineering, University of California, Berkeley, CA, USA
}

Scanning diffraction methods experiments in transmission electron microscopy have undergone substantial growth in recent years, due in part to the availability of high speed pixelated detectors with reasonable dynamic range. This capability enables new experiments that combine the benefits of position averaged convergent beam electron (PACBED) [1] with accurate partitioning of diffraction data into precise unit cell bins. A recent example of this combined technique is the quantitative determination of composition at projected unit cell resolution in $\mathrm{SrTiO}_{3}-\mathrm{La}$ 0.7 $\mathrm{Sr} 0 . \mathrm{MnO}_{3}$ multilayers from purely elastic scattering (Figure 1) [2]. PACBED has also been shown to be sensitive to the direction of polarization [3] and degree of octahedral tilt [4]. However, the optimal experimental conditions and limits of precision for mapping these quantitates quantitatively at unit cell resolution have yet to be fully explored.

We have initially focused on two model systems to independently address the quantitative error in measurements of polarization and octahedral rotation at single projected unit cell resolution. $\mathrm{BaTiO}_{3}$ films have been grown on $\mathrm{NdScO}_{3}$ (110), $\mathrm{SmScO}_{3}$ (110), $\mathrm{GdScO}_{3}$ (110) substrates using epitaxial strain to vary the degree of polarization from $\sim 25 \mu \mathrm{C} / \mathrm{cm}^{2}$ to $\sim 35 \mu \mathrm{C} / \mathrm{cm}^{2}$. In addition, $\mathrm{SrRuO}_{3}$ films have been grown on $\mathrm{SrTiO}_{3}$ substrates with and without a buffer layer of $\mathrm{GdScO}_{3}$ to modulate the degree of octahedral rotation in the surface film (Figure 2) [5], where the degree of rotation is expected to decay within a few unit cells from the interface. In order to verify the quantitative accuracy of these methods and determine optimal experimental parameters, we have performed a large set of PACBED simulations using implementations of the multislice [6] and $\mu$ STEM [7] methods. The use of machine learning algorithms for both data analysis and to facilitate lossless data compression will also be discussed [8].

References:

[1] J.M. LeBeau, S.D. Findlay, L.J. Allen et al, Ultramicroscopy, 110 (2010) p. 118

[2] C. Ophus, P. Ercius, M. Huijben, and J. Ciston, Applied Physics Letters, 110 (2017) p. 063102.

[3] J.M. LeBeau, A.J. D’Alfonso, N.J. Wright et al, Applied Physics Letters, 98 (2011) p. 052904.

[4] J. Hwang, J.Y. Zhang, J. Son et al, Applied Physics Letters, 100 (2012) 191909

[5] R. Gao, Y. Dong, H. Xu et al, Applied Materials and Interfaces, 8 (2016) p. 14871.

[6] E.J. Kirkland in "Advanced computing in electron microscopy", (Springer Science \& Business Media, 2010).

[7] L.J. Allen, A.J. D’Alfonso, and S.D. Findlay, Ultramicroscopy 151 (2015), p. 11.

[8] Work at the Molecular Foundry was supported by the Office of Science, Office of Basic Energy Sciences, of the U. S. Department of Energy under Contract No. DE-AC02-05CH11231. JC, YM, and RdR acknowledge additional support from the U. S. Department of Energy Early Career Research Program. 


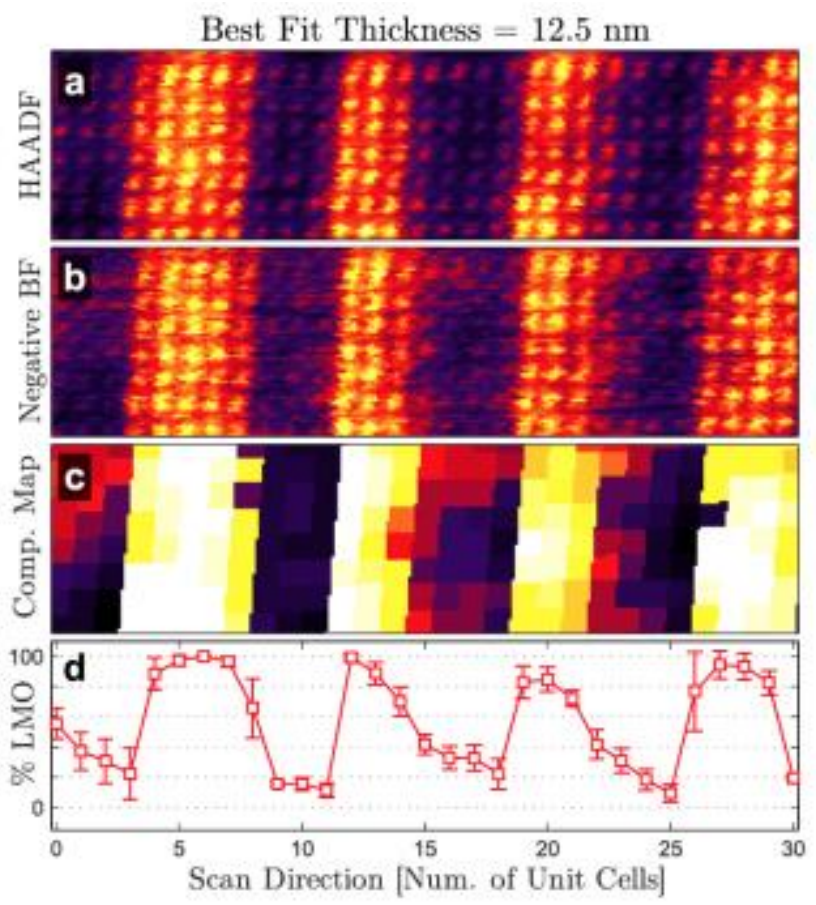

Figure 1. Elastic scattering composition measurements of $(\mathrm{LaMn})_{x}(\mathrm{SrTi})_{1-\mathrm{x}} \mathrm{O}_{3}$ a) HAADF image b) inverse of reconstructed bright-field c) composition maps at unit cell resolution from best-fit matches to PACBED simulations d) composition averaged perpendicular to the growth direction
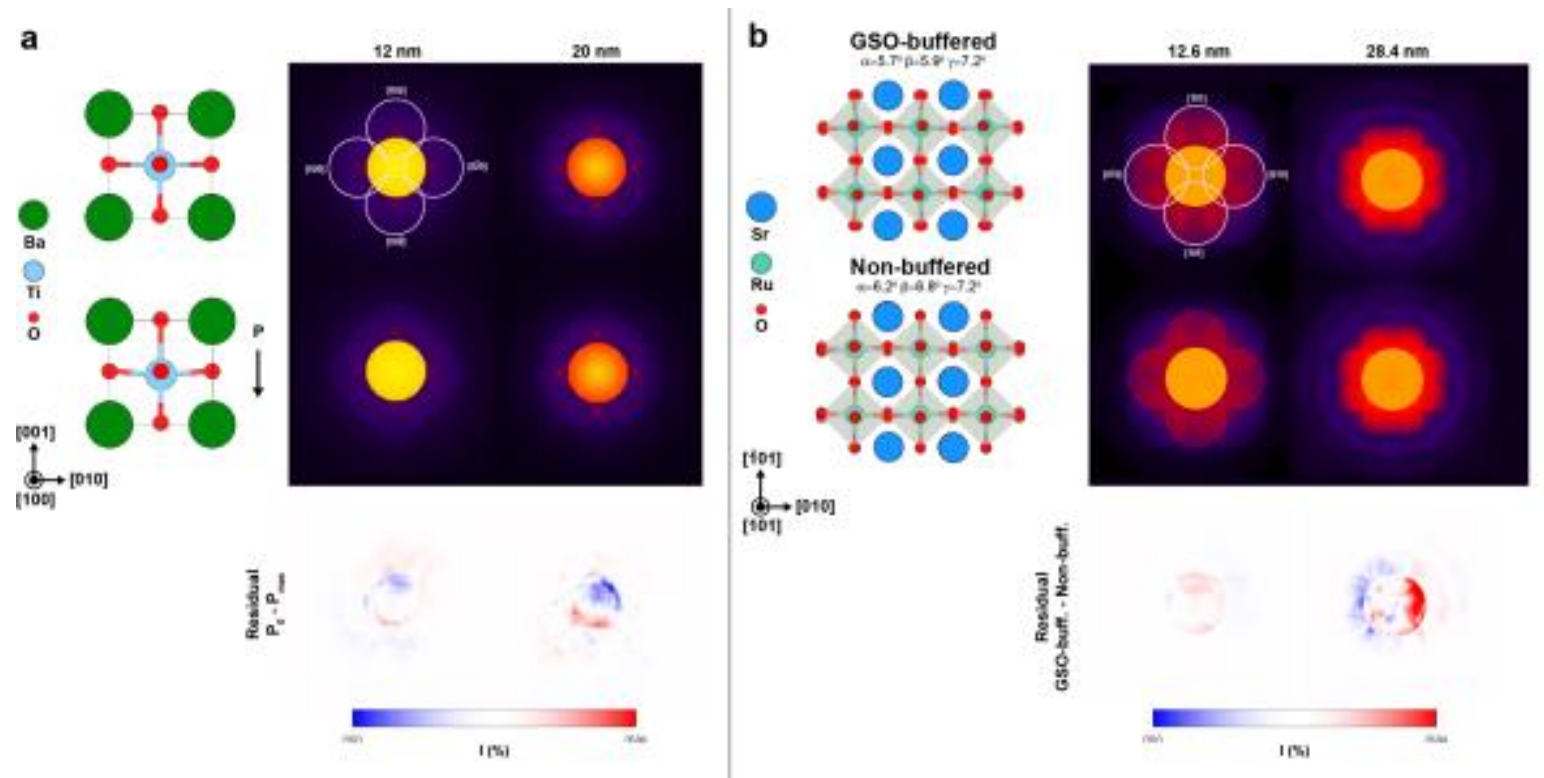

Figure 2. a) Structural model of $\mathrm{BaTiO}_{3}$ in the centrosymmetric and polarized $\left(35 \mu \mathrm{C} / \mathrm{cm}^{2}\right)$ states. PACBED simulations shown at right for $300 \mathrm{kV}$ electrons at $10 \mathrm{mrad}$ semi-convergence for $12 \mathrm{~nm}$ and $20 \mathrm{~nm}$ sample thickness. Difference maps plotted from $-0.6 \%$ to $0.6 \%$ of the incident probe intensity. b) Structural model of $\mathrm{SrRuO}_{3}$ film as grown on a $10 \mathrm{~nm}$ thick $\mathrm{GdScO}_{3}$ buffer layer (top) and pure $\mathrm{SrTiO}_{3}$ substrate (bottom). PACBED simulations shown at right for $300 \mathrm{kV}$ electrons at $10 \mathrm{mrad}$ semiconvergence for $12.6 \mathrm{~nm}$ and $28.4 \mathrm{~nm}$ sample thickness. Difference maps plotted from $-0.3 \%$ to $0.3 \%$ of the incident probe intensity. 